\title{
Estado de avance de la salud de los trabajadores en Perú
}

\section{Current status on the progress of workers' health in Peru}

Correspondencia César Cano Candiotti cesar.cano.c@upch.pe

Recibido: $19 / 03 / 2018$ Aprobado: 21/03/2018

Citar como: Cano Candiotti C, Francia Romero J. Estado de avance de la Salud de los trabajadores en Perú. Acta Med Peru. 2018;35(1):3-5

\author{
César Cano Candiotti ${ }^{1, a}$, José Francia Romero ${ }^{1, a}$ \\ 1 Unidad de Medicina Ocupacional y Medio Ambiente, Universidad Peruana Cayetano Heredia. Lima, Perú \\ a Especialista en Medicina Ocupacional y Medio Ambiente
}

Desde su inicio, la actividad laboral del ser humano ha permitido transformar el mundo, aunque también ha generado riesgos y enfermedades relacionadas al trabajo ${ }^{[1]}$. El dinamismo económico social y las nuevas formas de trabajo han introducido nuevos riesgos y enfermedades ocupacionales emergentes ${ }^{[2]}$ siendo su vigilancia continua, conocimiento y abordaje indispensables para alcanzar la salud de los trabajadores.

La salud ocupacional, rama de la salud pública, constituye uno de los cimientos fundamentales en el desarrollo de un país. El abordaje de la "salud del trabajador", en un contexto integral y multidisciplinario, no sólo analiza los hechos mórbidos que ocurren a razón de las condiciones de trabajo y riesgos ocupacionales sino también las condiciones en las cuales se vive y se trabaja ${ }^{[2]}$.

La población trabajadora constituye la mitad de la población del mundo ${ }^{[3]}$. Se estima que la carga global de enfermedad por accidentes y enfermedades relacionadas al trabajo es de 2,78 millones de muertes, lo que representa el $5 \%$ del total de muertes a nivel mundial, que en su mayoría corresponden a enfermedades relacionadas al trabajo $(86,3 \%$ ) y accidentes mortales $(13,7 \%)$; es decir, cada día mueren más de 7500 personas a causa de accidentes o enfermedades relacionadas al trabajo ${ }^{[4]}$.

Según la Organización Internacional del Trabajo (OIT) se estima que los costos directos e indirectos de las enfermedades y accidentes ocupacionales equivalen al $4 \%$ del producto bruto interno o 2,8 billones de dólares ${ }^{[5]}$.

Según el Instituto Nacional de Estadística e Informática peruano, para el 2015, la población en edad de trabajar era de 23034249 habitantes, de los cuales 16498138 (71,6\%) constituían la población económicamente activa (PEA) ${ }^{[6]}$. La economía está basada principalmente en la producción agrícola y la explotación minera ${ }^{[7]}$, y la distribución de la PEA ocupada por actividad económica presenta mayor proporción en servicios no personales, agricultura, ganadería, caza, silvicultura y comercio ${ }^{\left[{ }^{[6]}\right.}$.

En relación a las condiciones de empleo, se estima que el $71 \%$ de los trabajadores laboran en condiciones de informalidad ${ }^{[8]}$, situación que condiciona a mayor riesgo ocupacional, desprotección social y exposición a poblaciones vulnerables ${ }^{[2]}$. Por otro lado, la notificación de accidentes laborales, incidentes peligrosos y enfermedades ocupacionales no tiene todavía un sistema integrado de información. Mejía et al., en un estudio que buscó determinar las tendencias de los accidentes y enfermedades laborales, concluyeron que para el periodo 2010-2014 se reportaron 54596 accidentes laborales no mortales, 674 accidentes mortales, 3432 incidentes y 346 enfermedades laborales, identificándose una falta de homogenización y cumplimiento en el proceso de notificación por las entidades responsables ${ }^{[9]}$. 
La salud ocupacional en el Perú tuvo un desarrollo importante desde 1940 con la creación del departamento de Higiene Industrial en el Ministerio de Salud Pública, Trabajo y Promoción Social, que posteriormente se transformaría en el Instituto Nacional de Salud Ocupacional, siendo reconocido como centro de formación e investigación en la región ${ }^{[1]}$.

En las últimas dos décadas hubo una evolución significativa de la normativa laboral y el desarrollo de la salud ocupacional en el país: pasó de un enfoque inicial principalmente recuperativo, realizado por empresas de alto riesgo (minería, construcción, hidrocarburos, entre otras), a uno preventivo que incluya a todos los sectores económicos mediante la promulgación en el año 2011 de la "Ley de Seguridad y Salud en el Trabajo" (Ley N ${ }^{\circ}$ 29783). No obstante, a más de cinco años de su aplicación, no se ha alcanzado su adecuada implementación en todos los sectores, por lo que es necesario revisar las lecciones aprendidas y corregir las prácticas inadecuadas que se han dado en su aplicación.

Ante esta situación local y mundial, debido a los retos y cambios que impone el mundo del trabajo actual y el impacto de la carga de enfermedad descrito anteriormente, las organizaciones internacionales como la Organización de Naciones Unidas, la Organización Mundial de la Salud (OMS) y la OIT han establecido objetivos y metas dirigidos a proteger la vida y promover la salud y el bienestar de los trabajadores ${ }^{[10]}$. Un ejemplo es el Plan de Acción sobre la Salud de los trabajadores de la OMS para el período 2015-2025 que ha puesto como prioridad la salud de los trabajadores en la agenda de los estados miembros a fin de brindar la atención integral a la salud de los trabajadores, mejorar los entornos de trabajo, aumentar los esfuerzos para promover la salud de los trabajadores y disminuir las desigualdades en salud ${ }^{[10]}$

Analizaremos en forma general el estado de avance del Perú como país miembro OMS que ha participado y se ha adherido al Plan de Acción sobre la Salud de los trabajadores en base a las líneas estratégicas de acción propuestas ${ }^{[10]}$.

\section{Elaborar y actualizar la legislación y los reglamentos técnicos sobre la salud de los trabajadores}

Ya se cuenta con la Ley General de Seguridad y Salud en el Trabajo, su reglamento y modificatorias que aplican a todos los sectores económicos y a nivel nacional, un Sistema Nacional de Seguridad y Salud con conformación tripartita (Estado, trabajadores y empleadores) y un marco normativo relacionado. Sin embargo, no se ha alcanzado un adecuado cumplimiento en todos los sectores. Así mismo, se requiere armonizar la legislación y normativa relacionada a la salud de los trabajadores y trabajadoras entre los organismos y ministerios correspondientes. En relación a la vigilancia de la salud de los trabajadores y normas específicas se requieren su revisión, actualización y sistematización. Desde el 2017 se cuenta con el Plan Nacional de Seguridad y Salud en el Trabajo 2017-2021 cuyos ejes de acción están alineados a los convenios y organismos internacionales, su desarrollo y cumplimiento debe ser prioritario ${ }^{[6,10]}$.

\section{Identificar, evaluar, prevenir y controlar las condiciones y exposiciones peligrosas en el trabajo}

La identificación y evaluación de las condiciones de trabajo y exposiciones peligrosas requiere atención por parte del Estado. Actualmente, se cuenta con normativa sectorial en minería, hidrocarburos, electricidad, pesquería, construcción entre otras; sin embargo, no se ha implementado normativa en el sector agrícola que representa el mayor porcentaje de la PEA. También es necesario ver las estrategias para abordar al sector de la economía informal (representa cerca del $71 \%$ de los trabajadores) ${ }^{[8]}$ cuya siniestralidad es presumiblemente significativa. Se debe fortalecer la implementación de programas de prevención e intervención de desórdenes músculo-esqueléticos, cáncer profesional, riesgos psicosociales y control de violencia en lugar de trabajo.

\section{Aumentar el acceso y la cobertura de los servicios de salud para los trabajadores}

Rantanen et al. estimaron una cobertura baja de los servicios de salud ocupacional (SSO, del inglés occupational health services) en el Perú (aproximadamente el $10 \%$ de cobertura reportada) ${ }^{[11]}$. En los últimos cinco años ha habido un crecimiento exponencial de los SSO para los trabajadores formales, que inicialmente se enfocaron en la realización de evaluaciones médicas ocupacionales y posteriormente en el establecimiento de SSO en las empresas; este crecimiento requiere estandarizar y supervisar su práctica por los organismos responsables, tomando como base el cumplimiento de los principios éticos, técnicos basados en evidencia científica, alineados a los estándares internacionales y de calidad.

La OMS enfatizó recientemente la revitalización de los servicios de atención primaria y la integración de servicios básicos de salud ocupacional en el sistema; la experiencia de otros países podría evaluarse y aplicarse a nuestra realidad ${ }^{[11]}$. Por otro lado, uno de cada 16 trabajadores de la PEA y uno de cada 10 trabajadores que realizan actividad de alto riesgo está asegurado ${ }^{[7]}$ : se debe revisar el listado de actividades de alto riesgo y a mediano plazo implementar el aseguramiento universal.

Uno de los principios básicos para el desarrollo de SSO de acuerdo al convenio $\mathrm{N}^{\circ} 161$ de la OIT es organizarlos con equipos multidisciplinarios, los mismos que requieren formación y competencias específicas para su ejercicio, trabajo que debería realizarse en conjunto con las universidades, colegios profesionales y sociedades científicas ${ }^{[2,10]}$.

\section{Promover la salud, el bienestar y el trabajo saludable en el lugar de trabajo}

Se ha descrito que una adecuada atención integral de la salud de los trabajadores mejora la productividad. En este sentido, se debería adoptar el modelo de gestión de entornos saludables en el lugar de trabajo propuesto por la OMS y desarrollar la aplicación efectiva basada en la evidencia de intervenciones prácticas en promoción de la salud, bienestar laboral y calidad de vida en el trabajo ${ }^{[10,12]}$. 


\section{Fortalecer la capacidad de diagnóstico, los sistemas de información, la vigilancia epidemiológica, y la investigación de enfermedades, los accidentes y las muertes de trabajo}

La adecuada gestión de la información es indispensable para la vigilancia de la salud del trabajador. Es necesario implementar un sistema integrado y homogéneo de información de notificación de accidentes, enfermedades y muertes en el trabajo. Así mismo, se deben desarrollar protocolos o guías de diagnóstico de enfermedades profesionales que faciliten su reconocimiento y promover la investigación a fin de generar conocimiento y evidencia para los responsables de políticas y toma de decisiones ${ }^{[2,10,11]}$.

Finalmente concluimos que la salud de los trabajadores debe ser una prioridad para todos los sectores y más para el sector salud; es necesario el trabajo en conjunto con las instituciones, sociedades científicas y organismos vinculantes de manera armonizada y consensuada para fortalecer los lineamientos propuestos teniendo como finalidad primordial la prevención, protección y promoción de la salud de los trabajadores y trabajadoras formales e informales en todos los rubros de actividad económica y regiones del país.

\section{REFERENCIAS BIBLIOGRÁFICAS}

1. Gastañaga MC. Salud ocupacional: historia y retos del futuro. Rev Peru Med Exp Salud Publica. 2012;29(2):177-8.

2. Pando M. Salud Ocupacional en Latinoamérica. Revista Colombiana de Salud Ocupacional [Internet]. 2011 [citado el 5 de marzo de 2018];2(3):1-2. Disponible en: http://revistasojs.unilibrecali.edu. co/index.php/rcso/article/view/42

3. Organización Mundial de la Salud. Salud de los trabajadores: Plan de acción Mundial [Internet]. 60a Asamblea Mundial de la Salud. Ginebra Suiza, 14 al 23 de mayo del 2007 [citado el 5 de marzo de 2018]. Disponible en: http://www.who.int/occupational_health/ WHO_health_assembly_sp_web.pdf
4. Paivi H, Jukka T, Tan Boon K. Global estimates of occupational accidents and work related illnesses. Singapore: Workplace Safety and Health Institute; 2017.

5. Takala J, Hämäläinen P, Saarela KL, Yun LY, Manickam K, Jin TW, et al. Global estimates of the burden of injury and illness at work in 2012. J Occup Environ Hyg. 2014:11(5):326-37.

6. Decreto Supremo N ${ }^{\circ}$ 005-2017 TR. Decreto Supremo que aprueba el Plan Nacional de Seguridad y Salud en el Trabajo 2017-2021. El Peruano. 17 de abril del 2017.

7. Cruz I, Huerta-Mercado R. Occupational Safety and Health in Peru. Ann Glob Health. 2015;81(4):568-75.

8. Belapatiño V, Grippa F, Perea Hugo. Perú: Informalidad laboral y algunas propuestas para reducirla [Internet]. Observatorio Económico del Perú. Lima: BBVA Research; 2017 [citado el 12 de diciembre de 2017]. Disponible en: https://www.bbvaresearch. com/wp-content/uploads/2017/01/Observatorio-informalidadlaboral1.pdf

9. Mejia CR, Cárdenas MM, Gomero-Cuadra R. Notificación de accidentes y enfermedades laborales al Ministerio de Trabajo. Perú 2010-2014. Rev Peru Med Exp Salud Publica. 2015;32(3):526-31.

10. Panamericana de la Salud, Organización Mundial de la Salud. Plan de Acción sobre la Salud de los trabajadores 2015-2025 [Internet]. Washington, DC: OPS; c2017 [citado el 12 de diciembre de 2017]. Disponible en http://iris.paho.org/xmlui/ bitstream/handle/123456789/33983/CD54_10Rev.\%201-spa. pdf?sequence $=1 \&$ isAllowed $=y$

11. Rantanen J, Lehtinen S, Valenti A, lavicoli S. A Global survey on occupational health services in selected international commission on occupational health (ICOH) member countries. BMC Public Health. 2017;17:787.

12. Organización Panamericana de la Salud, Organización Mundia de la Salud. Ambientes de trabajo saludables: un modelo para la acción. Para empleadores, trabajadores autoridades normativas y profesionales. 2010 [Internet]. Washington, DC: OPS; c2017 [citado el 12 de diciembre de 2017]. Disponible en http://www.who.int/ phe/publications/healthy_workplaces/es/ 